\title{
5p13.3p13.2 duplication associated with developmental delay, congenital malformations and chromosome instability manifested as low-level aneuploidy
}

Ivan Y. Iourov ${ }^{1,2,3^{*}}$, Svetlana G. Vorsanova ${ }^{1,2}$, Irina A. Demidova ${ }^{1,2}$, Galina A. Aliamovskaia², Elena S. Keshishian² and Yuri B. Yurov ${ }^{1,2}$

\begin{abstract}
Recent developments in molecular cytogenetics allow the detection of genomic rearrangements at an unprecedented level leading to discoveries of previously unknown chromosomal imbalances (zygotic and post-zygotic) mosaic). These can be accompanied by a different kind of pathological genome variations, i.e. chromosome instability (CIN) manifested as structural chromosomal rearrangements and low-level mosaic aneuploidy. Fortunately, combining whole-genome and single-cell molecular cytogenetic techniques with bioinformatics offers an opportunity to link genomic changes to specific molecular or cellular pathology. High-resolution chromosomal SNP microarray analysis was performed to study the genome of a 15-month-aged boy presented with developmental delay, congenital malformations, feeding problems, deafness, epileptiform activity, and eye pathology. In addition, somatic chromosomal mutations (CIN) were analyzed by fluorescence in situ hybridization (FISH). Interstitial 5p13.3p13.2 duplication was revealed in the index patient. Moreover, CIN manifested almost exclusively as chromosome losses and gains (aneuploidy) was detected. Using bioinformatic analysis of SNP array data and FISH results, CIN association with the genomic imbalance resulted from the duplication was proposed. The duplication was demonstrated to encompass genes implicated in cell cycle, programmed cell death, chromosome segregation and genome stability maintenance pathways as shown by an interactomic analysis. Genotype-phenotype correlations were observed, as well. To the best our knowledge, identical duplications have not been reported in the available literature. Apart from genotypephenotype correlations, it was possible to propose a link between the duplication and CIN (aneuploidy). This case study demonstrates that combining SNP array genomic analysis, bioinformatics and molecular cytogenetic evaluation of somatic genome variations is able to provide a view on cellular and molecular pathology in a personalized manner. Therefore, one can speculate that similar approaches targeting both interindividual and intercellular genomic variations could be useful for a better understanding of disease mechanisms and disease-related biological processes.
\end{abstract}

Keywords: 5 p13 duplication, Chromosome instability, Aneuploidy, Mosaicism, Bioinformatics

\section{Background}

During the last decade, molecular cytogenetics has made significant efforts towards the development of new technologies, which are able to detect genomic

\footnotetext{
${ }^{*}$ Correspondence: ivan.iourov@gmail.com

${ }^{1}$ Mental Health Research Center, Russian Academy of Medical Sciences,

Moscow 117152, Russia

Full list of author information is available at the end of the article
}

rearrangements at an unprecedented resolution (Yan et al. 2009; Carvill and Mefford 2013; Kloosterman and Hochstenbach 2014; Riegel 2014). As a result, a broad spectrum of previously unreported microdeletion and microduplication syndromes has been discovered (Weise et al. 2012; Carvill and Mefford 2013). Additionally, numerous unique chromosomal (subchromosomal) abnormalities have been described providing important

\section{望 Springer}


clues regarding the origins and consequences of genomic rearrangements (Kloosterman and Hochstenbach 2014; Riegel 2014). Interestingly, both single gene mutations and copy number variations of chromosomal DNA (deletions/duplications) affecting almost all cells of the body (non-mosaic/heritable genetic changes), are able to produce genome or chromosome instability (CIN) manifesting as structural or numerical chromosomal changes (Thompson et al. 2010; Yurov et al. 2011; Forsberg et al. 2013; Heng et al. 2013; Iourov et al. 2013b; Pine and Liu 2014). CIN leading to the propagation of aneuploid cells represents an important phenomenon, which mediates intercellular genetic diversity in health and disease hallmarking cancers, neurodegeneration and aging (Michor et al. 2005; Iourov et al. 2009; Thompson et al. 2010; Vorsanova et al. 2010a; Yurov et al. 2011; Abdallah et al. 2013; Forsberg et al. 2013; Hultén et al. 2013). In this context, descriptions of cases featured by non-mosaic genetic changes and exhibiting increased levels of somatic mutations or CIN seem to be of fundamental importance (Michor et al. 2005; Iourov et al. 2013b).

Here, we report a male child with developmental delay, severe congenital malformations and interstitial duplication at 5p13.3p13.2, who has also exhibited increased levels of sporadic aneuploidy (CIN). Application of highresolution chromosomal SNP microarray analysis, fluorescence in situ hybridization (FISH) and bioinformatics has allowed speculations about correlations between genotype and phenotype. Furthermore, interactomic analysis has shown that CIN is likely to be associated with the duplication through a slight change of cell cycle, chromosome segregation, programmed cell death and genome stability maintenance pathways.

\section{Case description}

The male patient was referred to the hospital at the age of 15 months because of a developmental delay, congenital malformations, blindness, and feeding problems (poor appetite and frequent regurgitation). The familial history is positive for chronic gastritis and congenital heart malformation (mother), extensive brain hemorrhage (cousin; died at the ninth day postpartum) on the maternal side and congenital lung malformation (uncle; died at the first day postpartum), pancreatic cancer (grandfather) on the paternal side. The patient was born to a primigravida at 34th week of gestation by cesarean section. The pregnancy was threatened by miscarriage and was complicated by a low grade fever and exacerbation of gastritis (14th-17th weeks). Ultrasound showed oligohydramnios and fetal growth delay. The parents were non-consanguineous. The birth weight was $1297 \mathrm{~g}$ and birth length was $38 \mathrm{~cm}$ (both significantly less than 3rd percentile for age). Apgar score was 1 at $1 \mathrm{~min}$ and 4 at $5 \mathrm{~min}$. He had several respiratory failures and required intubation and intermittent positive pressure ventilation for 20 days. A diffuse hemorrhagic rash was noted at the first days of life. He had feeding problems, i.e. regurgitations persisting over a long period of time. Growth retardation was observed during the first year of life (always less than 3rd percentile for age). A developmental delay was also noted. The child did not recognize mother's face and was uncommunicative. Emotional response and object interests were lacking. At the age of 1 year, sensorineural deafness was revealed. Focal epileptiform activity in the left parietal-occipital region was also reported.

Physical examination at 15 months revealed a significant deficit in weight and decreased head circumference (less than 3rd percentile). He presented with dolichomicrocephaly, high forehead, ocular hypotelorism (the inner and outer canthal distances were both less than 3rd percentile), epicanthus, long philtrum, low set auricles, and enamel hypoplasia. Mild left-sided spastic hemiparesis and muscular hypotonia were noted. The psychomotor development (CAT/CLAMS scale) corresponded to the age of 5-6 months; the developmental coefficients were less than $50 \%$. He demonstrated stereotypic tapping of his head by his arm. The abdominal ultrasound examinations showed mild splenomegaly and increased echogenicity of parenchyma. The ophthalmological investigation showed descending optic atrophy, concomitant strabismus, nystagmus, zonular cataract of the left eye. Corpus callosum hypoplasia was uncovered by magnetic resonance imaging.

Cytogenetic analysis was performed by GTG- $(\sim 500$ band resolution) and C-banding and showed the presence of CIN (mainly chromosome gains or losses-aneuploidy). Constitutional chromosomal imbalances were not detected. Parental karyotypes were normal. To determine CIN rates, we performed FISH with DNA probes for chromosomes 1, 7, 16, 17, 18, $\mathrm{X}$ and $\mathrm{Y}$ as described earlier (Yurov et al. 2007; Iourov et al. 2009; Vorsanova et al. 2010b). FISH analysis confirmed the presence of CIN (aneuploidy) and showed that the rate of somatic/sporadic chromosomal mutations is 3-7 times higher in the index patient (Fig. 1) as to average chromosomal mutation rates in the general population according to our previous studies (Yurov et al. 2007; Iourov et al. 2009; Vorsanova et al. 2010a; Hultén et al. 2013).

High-resolution chromosomal SNP microarray analysis was performed on DNA isolated from peripheral blood lymphocytes using CytoScan ${ }^{\circledR}$ HD Arrays (Affymetrix, Santa Clara, CA) consisting of $\sim 2.7$ million markers for CNV evaluation and 750,000 SNPs and the Affymetrix Chromosome Analysis Suite software as described earlier (Iourov et al. 2012, 2013a; Wang et al. 2014). Genomic 

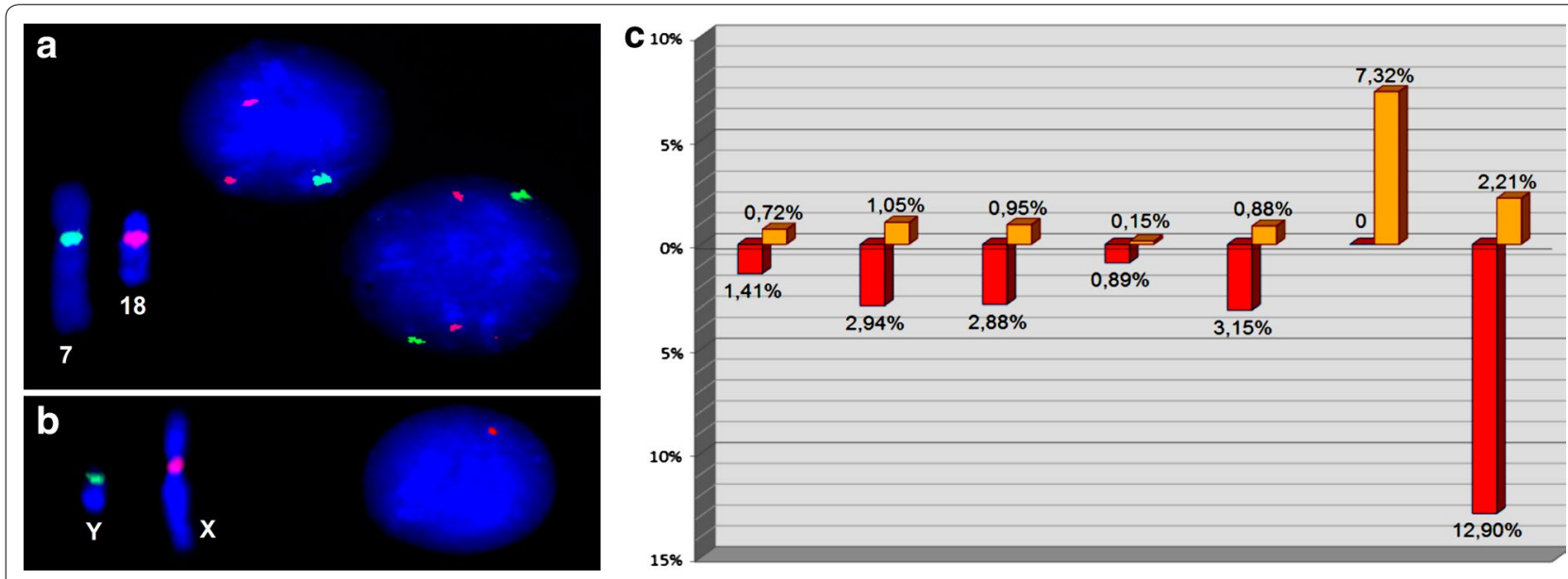

Fig. 1 Interphase FISH analysis of CIN (somatic aneuploidy). a FISH with DNA probes for chromosomes 7 (green) and 18 (red) showing chromosome 7 loss in the right nucleus (metaphase chromosomes show positive signals for these DNA probes). b Interphase FISH with DNA probes for chromosomes $Y$ (green) and X (red) showing chromosome Y loss in the nucleus (metaphase chromosomes show positive signals for these DNA probes). c Rates of chromosome losses (red bars) and gains (golden bars)

localization and gene content was defined using NCBI Build GRCh37/hg19 reference sequence. Genomic copy number variations were further addressed by a bioinformatic analysis described recently (Iourov et al. 2014). Interactomic analysis was performed using STRING v9.1 online tool (Franceschini et al. 2013). High-resolution chromosomal SNP microarray analysis has detected a duplication located at 5p13.3p13.2 ( 994 kbps) spanning 11 genes, 6 of which have assigned functions and are listed in OMIM (Online Mendelian Inheritance in Men) database (http://omim.org/) (Fig. 2). The duplication was de novo.

According to microarray analysis (Fig. 2), 6 genes were found to be eligible for bioinformatic evaluation. TARS (OMIM:\#187790) encodes an aminoacyl-tRNA synthetase interacting with numerous proteins from this family. Secondary functions of TARS are shown to be associated with the nervous system-related disorders and autoimmune diseases (Williams et al. 2013). ADAMTS12 (OMIM:\#606184) encodes a member of a disintegrin and metalloproteinase with thrombospondin motifs protein family with tumour-protective functions
(Llamazares et al. 2007) interacting with a number of gene products, among which there are proteins implicated in Notch signaling pathway (NOTCH1, FURIN). Functionally, ADAMTS12 copy number variation has the potential to be relevant to numerous pathogenic processes including those associated with brain dysfunction, autoimmunity, inflammation, and cancer. RXFP3 (OMIM:\#609445) is a member of relaxin family peptide receptors. Bioinformatic analysis shows that copy number variation of $R X F P 3$ may alter neuropeptide signaling processes, organization of neuroendocrine signals and stimulation of water drinking and food intake. SLC45A2 (OMIM:\#606202) encodes a transporter protein mediating melanin synthesis and is mutated in oculocutaneous albinism type IV (OMIM:\#606574). According to BioGPS, this gene is expressed in retina (http://biogps.org/\#got $\mathrm{o}=$ genereport\&id=51151). AMACR (OMIM:\#604489) encodes a racemase and is likely to be mutated in adultonset sensorimotor neuropathy, pigmentary retinopathy, and adrenomyeloneuropathy due to defects in bile acid synthesis. In addition, the gene product interacts with multifunctional proteins, which play a role in

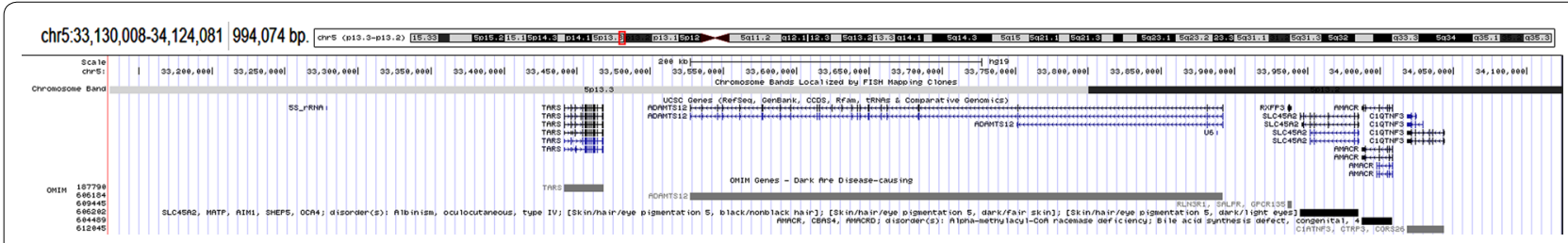

Fig. 2 Schematic representation of 5p13.3p13.2 duplication detected by high-resolution chromosomal microarray analysis displayed using UCSC Genome Browser on Human Feb. 2009 Q2 381 (GRCh37/hg19) Assembly (http://genome-euro.ucsc.edu/index.html) 
transcriptional and cell cycle regulation, programmed cell death, and genome stability maintenance (Lloyd et al. 2012). C1QTNF3 (OMIM:\#612045) is a gene encoding $\mathrm{C} 1 \mathrm{q}$ and tumor necrosis factor related protein 3 mutated in neurobehavioral diseases. The gene product interacts with leptin, an element of a signaling pathway that acts to regulate the size of the body, fat depot and may act on the brain to inhibit food intake. Bioinformatic analysis has given rise to speculations that this duplication is likely to be causative for developmental delay and congenital malformations in the index case. Moreover, it allowed us to determine the molecular pathway to CIN, the elements of which were involved in the duplication.

\section{Discussion and evaluation}

During the last decades, there have been several reports on duplications at 5p13 (Lorda-Sánchez et al. 1997; Cervera et al. 2005; Loscalzo et al. 2008). Further molecular cytogenetic studies have evidenced that similar duplications cause chromosome 5p13 duplication syndrome (OMIM \#613174) involving the NIPBL gene. Clinically, the syndrome is characterized by developmental delay and intellectual disability with facial dysmorphism featured by frontal bossing, large or broad forehead, bitemporal narrowing, short/slanted palpebral fissures, short philtrum, high-arched palate, and low-set ears (Yan et al. 2009). However, the index case has not demonstrated duplication of the critical region of the chromosome 5 p13 duplication syndrome. Nevertheless, molecular and clinical overlap between classical chromosome 5p13 duplication syndrome and the index case was noted. Interestingly, two cases (patients: 286710 and 300639) from DECIPHER v8.8 (https://decipher.sanger.ac.uk) exhibited duplications encompassing shortly genomic loci affected by the duplication in the index patient. Although patient 300639 presented with global developmental delay, both cases seem to demonstrate a milder phenotype compared to the present case. Nonetheless, it is to note that these cases were not identical to the present one. The phenotypic differences are likely to be explained by incomplete overlap of duplicated chromosomal DNA sequences between DECIPHER cases and the index patient. To provide case-specific genotype-phenotype correlations, we have used a recently described bioinformatic algorithm for molecular cytogenetic analysis (Iourov et al. 2014).

Bioinformatic evaluation of duplicated genes enabled us to speculate on genotype-phenotype correlations. Thus, copy number increase of TARS is likely to be related to developmental delay and congenital malformations; ADAMTS12-brain dysfunction, developmental delay and congenital malformations; $R X F P 3$ - neurological symptoms and feeding problems; SLC45A2-eye pathology; $A M A C R$ - neurological symptoms (i.e. deafness), eye pathology and congenital malformations; C1QTNF3-growth retardation and feeding problems. Although in silico evaluation allowed genotype-phenotype correlations in the same fashion as done for almost all contiguous gene deletion/duplication syndromes (Yan et al. 2009; Weise et al. 2012; Kloosterman and Hochstenbach 2014; Carvill and Mefford 2013), there can be a certain bias and cross effects between phenotypic contribution of each duplicated gene. Surprisingly, the phenotype of the duplication was rather severe. Still, bioinformatics analysis, genotype-phenotype correlations and comparative evaluation with previous reports on chromosome 5 p13 duplication syndrome do indicate a possible causative role of this genomic rearrangement.

It is noteworthy that ADAMTS12, AMACR, SLC45A2 and C1QTNF3 are associated with cancers, which are all hallmarked by CIN (aneuploidy) (Michor et al. 2005; Llamazares et al. 2007; Lloyd et al. 2012; Abdallah et al. 2013). Somatic aneuploidy is generally a result of alterations to cell cycle checkpoint, chromosome segregation, programmed cell death and genome stability maintenance pathways, which can be fused into a single generalized "aneuploidization" pathway (Harris and Levine 2005; Iourov et al. 2009, 2013b; Yurov et al. 2011; Abdallah et al. 2013; Heng et al. 2013). Interactome analysis of proteins encoded by duplicated genes provided a possibility to link the genomic rearrangement and CIN (Fig. 3). It has been observed that ADAMTS12 and AMACR interactomes are interconnected via NOTCH1 and TP53. These are key elements of global pathways of cell cycle/ fate and transcriptional activity regulation. Furthermore, NOTCH1 and TP53 are together involved in pathways leading to CIN in a variety of cancers (Roemer 2012; Pine and Liu 2014). According to the analysis, the connection between ADAMTS12 and AMACR interactomes implicate numerous elements of the critical pathways, which were previously grouped under the umbrella term "aneuploidzation" pathway (Iourov et al. 2009, 2013b; Yurov et al. 2011; Abdallah et al. 2013; Heng et al. 2013). In this light, it is to mention MAPK14 as an element of SLC45A2 interactome (Fig. 3), inasmuch as MAPK14 gene product has been shown to play a role in progression of TP53(-/-) cells exhibiting numerical chromosome abnormalities and CIN (Vitale et al. 2010). It is to recognize that duplicated genes are indirectly involved in the aforementioned pathway. Taking this fact into account, we admit that CIN might be a result of another genetic or epigenetic change. However, since CIN in the index patient is limited to increase of somatic aneuploidy rates being significantly milder than in a cell population exhibiting alterations to the genes directly implicated in the aforementioned pathway (i.e. TP53, EP300, SP1 etc.) 


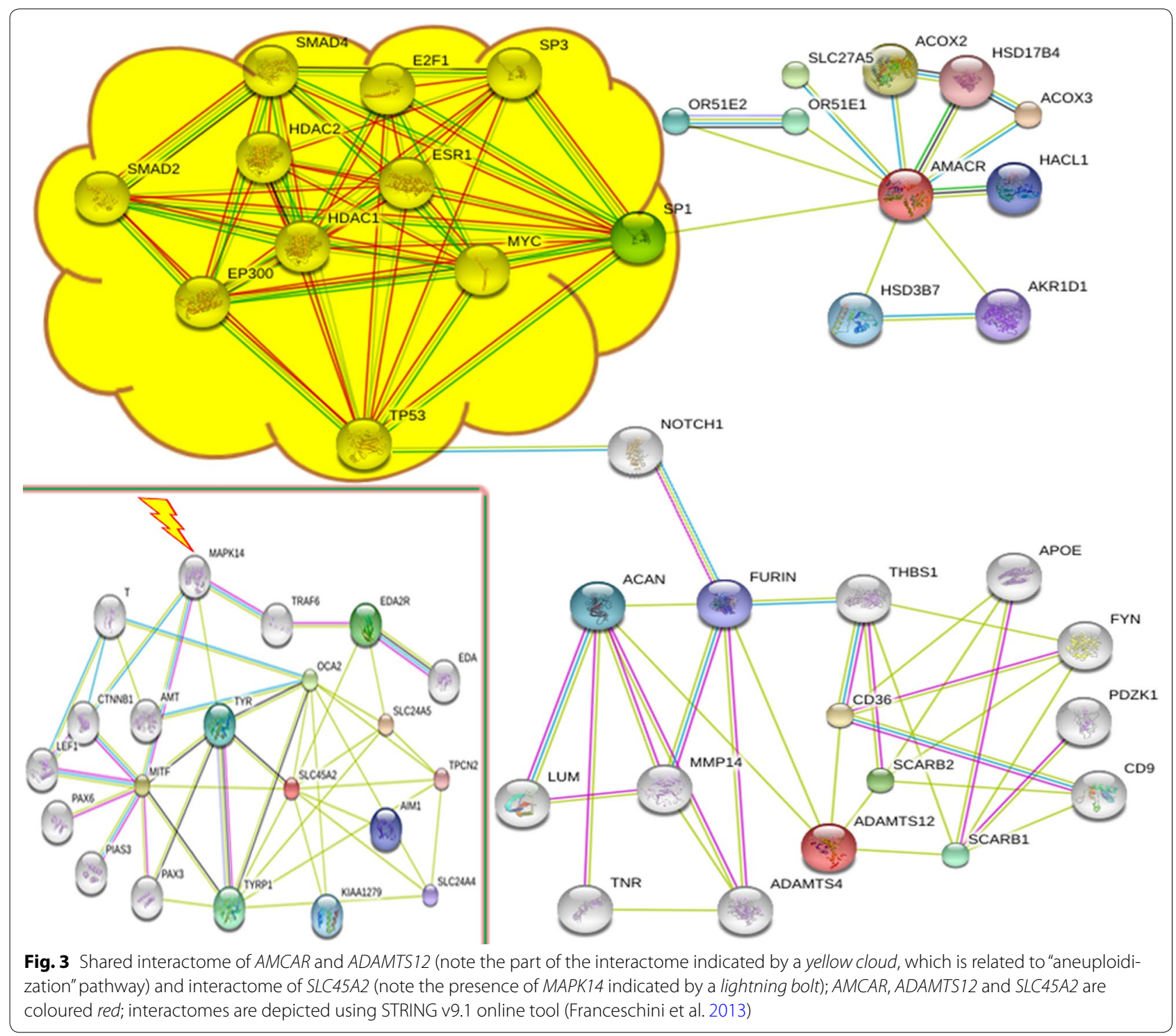

(Thompson et al. 2010; Roemer 2012; Heng et al. 2013; Pine and Liu 2014). Consequently, we have hypothesized that alterations to less essential neighbors in an interactome network are more likely to result into a slight modification of the pathway. The latter can produce specific types of CIN without an immediate impact on cellular genotype/phenotype. Additionally, CIN affecting detectable cell populations should be considered a potential risk factor for disorders mediated by genome instability (i.e. cancer and neurodegeneration) requiring molecular cytogenetic monitoring of somatic chromosomal mutations. To this end, we have to state that a relationship between CIN and 5p13.3p13.2 duplications requires additional case-reports as well as additional molecular analyses.

\section{Conclusion}

In the present case study, a description of 5p13.3p13.2 duplication in a child with developmental delay, congenital malformations and CIN (sporadic aneuploidy) is given. Using high-resolution chromosomal SNP microarray analysis, FISH evaluation of somatic aneuploidy and bioinformatics, we were able to propose genotypephenotype correlation and to hypothesize a link between non-mosaic 5p13 duplication and sporadic mosaic aneuploidy (CIN) in the index case. Accordingly, we speculate that combining techniques targeting interindividual and intercellular genome variations together with bioinformatic analyses is able to uncover underlying basis of phenotypic changes due to genomic rearrangements or disease mechanisms in a personalized manner. 


\section{Abbreviations}

CIN: chromosome instability; FISH: fluorescence in situ hybridization; SNP: single nucleotide polymorphism.

\section{Authors' contributions}

$I Y I, S G V$ and YBY conceived the research, designed the study, wrote the manuscript, and obtained the funding. IYI and IAD performed the experiments. GAA and ESK referred the patient for the study. All authors have read and approved the final manuscript.

\section{Author details}

${ }^{1}$ Mental Health Research Center, Russian Academy of Medical Sciences, Moscow 117152, Russia. ${ }^{2}$ Russian National Research Medical University named after N.I. Pirogov, Separated Structural Unit "Clinical Research Institute of Pediatrics", Ministry of Health of Russian Federation, Moscow 125412, Russia. ${ }^{3}$ Department of Medical Genetics, Russian Medical Academy of Postgraduate Education, Moscow 123995, Russia.

\section{Acknowledgements}

We thank Oxana S. Kurinnaia, Maria A. Zelenova and Kirill S Vasin for technical assistance. This study makes use of data generated by the DECIPHER Consortium; a full list of centers who contributed to the generation of the data is available from http://decipher.sanger.ac.uk and via email from decipher@ sanger.ac.uk (funding for the DECIPHER project was provided by the Wellcome Trust). The study was supported by the Russian Science Foundation (project \#14-15-00411).

\section{Competing interests}

The authors declare that they have no competing interests.

\section{Received: 13 May 2015 Accepted: 5 October 2015}

Published online: 15 October 2015

\section{References}

Abdallah BY, Horne SD, Stevens JB, Liu G, Ying AY, Vanderhyden B, Krawetz SA, Gorelick R, Heng HH (2013) Single cell heterogeneity: why unstable genomes are incompatible with average profiles. Cell Cycle 12:3640-3649

Carvill GL, Mefford HC (2013) Microdeletion syndromes. Curr Opin Genet Dev 23:232-239

Cervera M, Sánchez S, Molina B, Alcántara MA, Del Castillo V, Carnevale A, González-del Angel A (2005) Trisomy of the short arm of chromosome 5 due to a de novo inversion and duplication (5)(p15.3 p13.3). Am J Med Genet A 136A:381-385

Forsberg LA, Absher D, Dumanski JP (2013) Non-heritable genetics of human disease: spotlight on post-zygotic genetic variation acquired during lifetime. J Med Genet 50:1-10

Franceschini A, Szklarczyk D, Frankild S, Kuhn M, Simonovic M, Roth A, Lin J, Minguez P, Bork P, von Mering C, Jensen LJ (2013) STRING v9.1: proteinprotein interaction networks, with increased coverage and integration. Nucleic Acids Res 41:D808-D815

Harris SL, Levine AJ (2005) The p53 pathway: positive and negative feedback loops. Oncogene 24:2899-2908

Heng HH, Bremer SW, Stevens JB, Horne SD, Liu G, Abdallah BY, Ye KJ, Ye CJ (2013) Chromosomal instability (CIN): what it is and why it is crucial to cancer evolution. Cancer Metastasis Rev 32:325-340

Hultén MA, Jonasson J, Iwarsson E, Uppal P, Vorsanova SG, Yurov YB, lourov IY (2013) Trisomy 21 mosaicism: we may all have a touch of Down syndrome. Cytogenet Genome Res 139:189-192

lourov IY, Vorsanova SG, Liehr T, Kolotii AD, Yurov YB (2009) Increased chromosome instability dramatically disrupts neural genome integrity and mediates cerebellar degeneration in the ataxia-telangiectasia brain. Hum Mol Genet 18:2656-2669

lourov IY, Vorsanova SG, Kurinnaia OS, Zelenova MA, Silvanovich AP, Yurov YB (2012) Molecular karyotyping by array CGH in a Russian cohort of children with intellectual disability, autism, epilepsy and congenital anomalies. Mol Cytogenet 5:46 lourov IY, Vorsanova SG, Voinova VY, Kurinnaia OS, Zelenova MA, Demidova IA, Yurov YB (2013a) Xq28 (MECP2) microdeletions are common in mutationnegative females with Rett syndrome and cause mild subtypes of the disease. Mol Cytogenet 6:53

lourov IY, Vorsanova SG, Yurov YB (2013b) Somatic cell genomics of brain disorders: a new opportunity to clarify genetic-environmental interactions. Cytogenet Genome Res 139:181-188

lourov IY, Vorsanova SG, Yurov YB (2014) In silico molecular cytogenetics: a bioinformatic approach to prioritization of candidate genes and copy number variations for basic and clinical genome research. Mol Cytogenet 7:98

Kloosterman WP, Hochstenbach R (2014) Deciphering the pathogenic consequences of chromosomal aberrations in human genetic disease. Mol Cytogenet 7:100

Llamazares M, Obaya AJ, Moncada-Pazos A, Heljasvaara R, Espada J, LópezOtín C, Cal S (2007) The ADAMTS12 metalloproteinase exhibits antitumorigenic properties through modulation of the Ras-dependent ERK signaling pathway. J Cell Sci 120:3544-3552

Lloyd MD, Yevglevskis M, Lee GL, Wood PJ, Threadgill MD, Woodman TJ (2012) $\alpha$-Methylacyl-CoA racemase (AMACR): metabolic enzyme, drug metabolizer and cancer marker P504S. Prog Lipid Res 52:220-230

Lorda-Sánchez I, Urioste M, Villa A, Carrascosa MC, Vázquez MS, Martínez A, Martínez-Frías ML (1997) Proximal partial 5p trisomy resulting from a maternal (19;5) insertion. Am J Med Genet 68:476-480

Loscalzo ML, Becker TA, Sutcliffe M (2008) A patient with an interstitial duplication of chromosome 5p11-p13.3 further confirming a critical region for $5 p$ duplication syndrome. Eur J Med Genet 51:54-60

Michor F, Iwasa Y, Vogelstein B, Lengauer C, Nowak MA (2005) Can chromosomal instability initiate tumorigenesis? Semin Cancer Biol 15:43-49

Pine SR, Liu W (2014) Asymmetric cell division and template DNA co-segregation in cancer stem cells. Front Oncol 4:226

Riegel M (2014) Human molecular cytogenetics: from cells to nucleotides. Genet Mol Biol 37:194-209

Roemer K (2012) Notch and the p53 clan of transcription factors. Adv Exp Med Biol 727:223-240

Thompson SL, Bakhoum SF, Compton DA (2010) Mechanisms of chromosomal instability. Curr Biol 20:R285-R295

Vitale I, Jemaà M, Senovilla L, Galluzzi L, Rello-Varona S, Metivier D, Ripoche H, Lazar V, Dessen P, Castedo M, Kroemer G (2010) Involvement of p38alpha in the mitotic progression of p53(-/-) tetraploid cells. Cell Cycle 9:2823-2829

Vorsanova SG, Yurov YB, Soloviev IV, lourov IY (2010a) Molecular cytogenetic diagnosis and somatic genome variations. Curr Genomics 11:440-446

Vorsanova SG, Yurov YB, lourov IY (2010b) Human interphase chromosomes: a review of available molecular cytogenetic technologies. Mol Cytogenet 3:1

Wang BT, Chong TP, Boyar FZ, Kopita KA, Ross LP, El-Naggar MM, Sahoo T, Wang JC, Hemmat M, Haddadin MH, Owen R, Anguiano AL (2014) Abnormalities in spontaneous abortions detected by G-banding and chromosomal microarray analysis (CMA) at a national reference laboratory. Mol Cytogenet 7:33

Weise A, Mrasek K, Klein E, Mulatinho M, Llerena JC Jr, Hardekopf D, Pekova S, Bhatt S, Kosyakova N, LiehrT (2012) Microdeletion and microduplication syndromes. J Histochem Cytochem 60:346-358

Williams TF, Mirando AC, Wilkinson B, Francklyn CS, Lounsbury KM (2013) Secreted Threonyl-tRNA synthetase stimulates endothelial cell migration and angiogenesis. Sci Rep 3:1317

Yan J, Zhang F, Brundage E, Scheuerle A, Lanpher B, Erickson RP, Powis Z, Robinson HB, Trapane PL, Stachiw-Hietpas D, Keppler-Noreuil KM, Lalani SR, Sahoo T, Chinault AC, Patel A, Cheung SW, Lupski JR (2009) Genomic duplication resulting in increased copy number of genes encoding the sister chromatid cohesion complex conveys clinical consequences distinct from Cornelia de Lange. J Med Genet 46:626-634

Yurov YB, Vorsanova SG, lourov IY, Demidova IA, Beresheva AK, Kravetz VS, Monakhov V, Kolotii AD, Voinova-Ulas VY, Gorbachevskaya NL (2007) Unexplained autism is frequently associated with low-level mosaic aneuploidy. J Med Genet 44:521-525

Yurov YB, Vorsanova SG, lourov IY (2011) The DNA replication stress hypothesis of Alzheimer's disease. Sci World J 11:2602-2612 